were integrated care/case management packages with significant self-management components. RCT follow-up ranged from 3-12 months with a total of 1113 (range 33-464) patients enrolled. Results from $\mathrm{n}=4$ RCTs indicate a reduction in re-admissions of borderline significance (OR 0.65 (95\% CI 0.42, 1.00)) but no significant effect on mortality (OR 1.22 (95\% CI $0.79,1.86)$ ). Effect on overall quality of life was heterogeneous with large loss-to-follow-up. There were no cost-effectiveness studies.

Conclusions There is a paucity of good quality large RCTs of supported self-management delivered at discharge. Interventions are disparate and few studies report significant benefits in important outcomes. However, effect sizes for reduction in admissions are consistent with published evidence of self-management interventions delivered whilst patients are stable.

\section{P47 PATIENT CHARACTERISTICS OF THOSE REFERRED AND NOT REFERRED FOR EARLY POST-HOSPITALISATION PULMONARY REHABILITATION}

${ }^{1}$ SE Jones, ${ }^{1} \mathrm{SSC}$ Kon, ${ }^{2} \mathrm{SA}$ Green, ${ }^{2} \mathrm{D}$ Bell, ${ }^{1} \mathrm{JL}$ Canavan, ${ }^{1} \mathrm{CM}$ Nolan, ${ }^{3} \mathrm{AL}$ Clark, ${ }^{4} \mathrm{M}$ Dickson, ${ }^{4} \mathrm{~A}-\mathrm{M}$ Nolan, ${ }^{1} \mathrm{~S}$ Fleming, ${ }^{4} \mathrm{M}$ Haselden, ${ }^{1} \mathrm{WD}-\mathrm{C}$ Man; ${ }^{1}$ Respiratory NIHR Biomedical Research Unit, Royal Brompton \& Harefield NHS Foundation Trust and Imperial College, Harefield, Middlesex, United Kingdom; ${ }^{2}$ NIHR CLAHRC for Northwest London, London, UK; ${ }^{3}$ Harefield Pulmonary Rehabilitation Unit, Royal Brompton and Harefield NHS Foundation Trust, Harefield, Middlesex, United Kingdon; ${ }^{4}$ The Hillingdon Hospital NHS Foundation Trust, Uxbridge, UK

\subsection{6/thoraxjnl-2013-204457.197}

Background Early post-hospitalisation pulmonary rehabilitation (PR) following acute exacerbation of COPD (AECOPD) has been shown to improve health-related quality of life (HRQOL), increase exercise capacity and reduce rate of hospital readmission. However, only a minority of eligible patients are referred for (or receive) this intervention. The aim of this study was to determine differences in baseline characteristics between those referred or not referred for early post-hospitalisation PR. We hypothesised that those with poorer lung function, worse functional capacity, increased muscle weakness and cachexia would be less likely to be referred for early post-hospitalisation PR.

Methods Two hundred and twenty six patients hospitalised for AECOPD were consecutively recruited on day of hospital discharge. All fulfilled the eligibility criteria for PR, which included the ability to walk 5 metres independently. The following measurements were performed on day of hospital discharge by the research team: spirometry, anthropometry (body mass index: BMI and fat free mass index (FFMI)), lower limb muscle strength (Quadriceps Maximum Voluntary Contraction: QMVC), functional capacity (4-metre gait speed (4MGS)), HRQOL (COPD Assessment Test (CAT)) and Hospital Anxiety and Depression scale (HAD)). Length of stay (LOS), previous admissions to hospital in past year, social deprivation scores (based on postcode) and smoking history were also recorded. The decision to refer was made by the clinical team, blinded to results of outcome measurements.

Results The results are seen in Table 1 . Seventy three patients (32\%) were referred for early post-hospitalisation PR. Contrary to our hypothesis, there was no difference in spirometry, muscle strength, functional capacity or muscle mass between patients that were referred or not referred for early post-hospitalisation PR. There were also no differences in HRQOL, anxiety or depression scores, smoking status, social deprivation score or number of hospitalisations in past year. The only significant difference was a slightly reduced length of hospital stay for those referred to PR

Conclusion Reasons for non-referral for post-hospitalisation PR cannot be simply explained by physiological characteristics at hospital discharge, and are likely to be secondary to complex interactions between patient and healthcare professionals. Further qualitative work is required to understand these interactions and relationships.

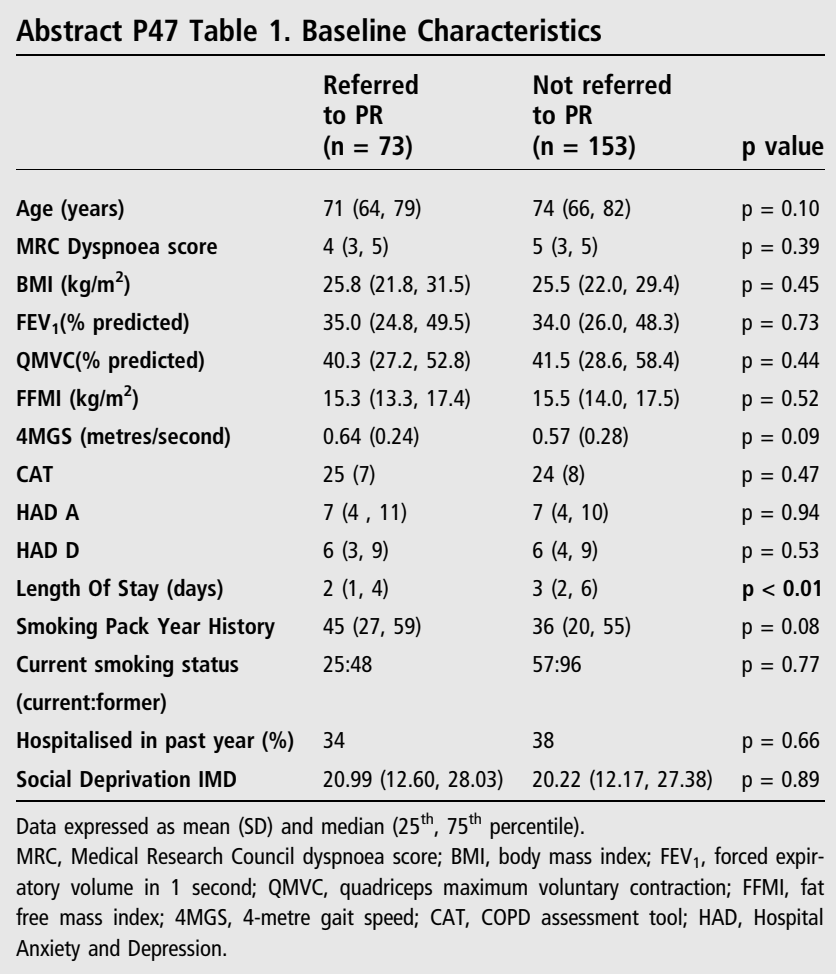

\section{P48 AN EVALUATION TO UNDERSTAND THE USE OF TECHNOLOGY WITHIN A COPD POPULATION}

${ }^{1} \mathrm{M}$ Gibb, 'V Willott, ${ }^{1} \mathrm{~S}$ Lohar, ${ }^{1} \mathrm{~S}$ Ward, ${ }^{2} \mathrm{C}$ Bolton, ${ }^{3} \mathrm{P}$ McAlinden, ${ }^{4} \mathrm{~A}$ De-Soyza, ${ }^{5} \mathrm{~S}$ Singh; ${ }^{1}$ University Hospital of Leicester, Leicester, United Kingdom; ' ${ }^{2}$ University of Nottingham, Nottingham, United Kingdom; ${ }^{3}$ Sir William Leech Centre for Lung Research Freeman Hospital, Newcastle Upon Tyne, United Kingdom; ${ }^{4}$ Institute of Cellular medicine, Newcastle University and COPD Service Freeman Hospital Newcastle, Newcastle Upon Tyne, United Kindom; ${ }^{5}$ Faculty of Health and Life Sciences Coventry University, Coventry, United Kingdom

\subsection{6/thoraxjnl-2013-204457.198}

Introduction and Objectives There is a desire to employ technology to support patients with long term conditions. However there is little data available that describes familiarity with technology in the COPD population. We have an interest in developing alternative forms of pulmonary rehabilitation deploying technology. Therefore the aim of this evaluation was to understand the use of technology in this population.

Methods Patients attending a consultant led COPD follow up clinic were asked to fill out a 10 itemed survey regarding their physical activity levels, if they had an interest in pulmonary rehabilitation and technological devices they may use.

Results 191 patients returned the surveys, 168 from the Glenfield Hospital Leicester and 23 from the Newcastle upon Tyne Hospitals. The population surveyed consisted of 76 males, 81 females and 34 who did not specify their gender. The age range 
of population was; <40 yrs 14 (8\%); 40-50 yrs 18 (9.6\%); 5060 yrs 29 (15.5\%); 60-70 yrs 61 (32.6\%); 70-80 yrs 47 (25.1\%); > 80yrs 17 (9.1\%). Within the total population it was found that $112(60.5 \%)$ owned a computer and $138(74.2 \%)$ own a mobile telephone however, of 138 only $22(11.8 \%)$ were owners of a smart phone. Within each age range there were a small number of smart phone users but the predominant usage of smart phones occurred within the younger age ranges. Furthermore, within each age range a higher percentage of the population owned a mobile phone than those that did not. Similarly within each age range a higher percentage of the population owned a computer than did not, excluding the $>80$ yrs, where 9 $(52.9 \%)$ did not own a computer.

Conclusion Overall the use of technology is limited in this COPD population. A significant proportion of those taking part used a mobile phone but a very small percentage used a smart phone, upon which a number of interventions might be delivered. Over half the population had a computer. There is a potential target market for providing alternative forms of pulmonary rehabilitation utilising technology, however, more evaluation is needed to ascertain whether a technological intervention would be acceptable to these patients.

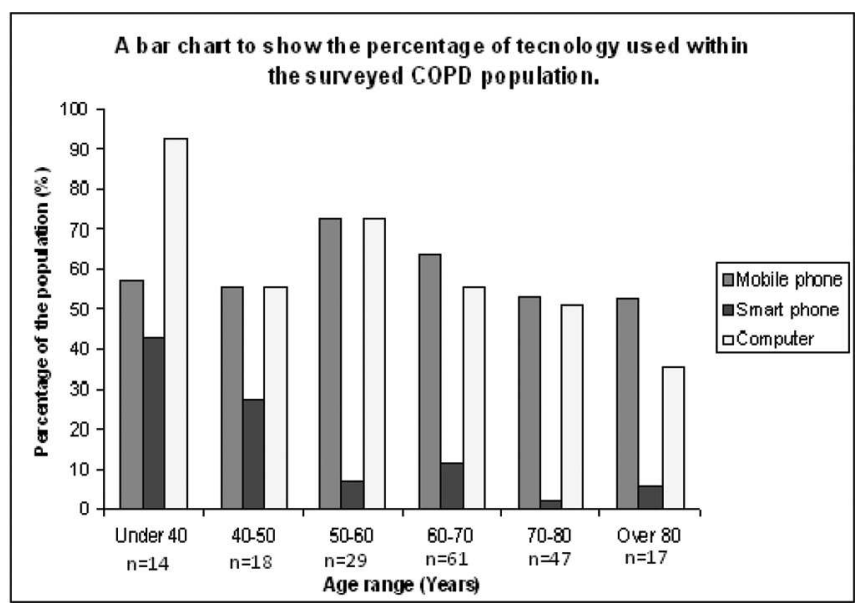

Abstract P48 Figure 1.

\section{Lung cancer: investigation and treatment}

\section{P49 INCIDENTAL PULMONARY NODULES; ARE WE DOING TOO MANY FOLLOW UP SCANS? SERVICE REVIEW AND VALUE OF PET-CT IMAGING}

${ }^{1} Z$ Zaitout, ${ }^{2} \mathrm{~A}$ Zia, ${ }^{2} \mathrm{R}$ Senasi, ${ }^{2}$ S Matthews; ${ }^{1}$ Barts Health, London, Greater London; ${ }^{2}$ Sheffield Teaching Hospitals, Sheffield, South Yorkshire

\subsection{6/thoraxjn-2013-204457.199}

Incidental lung nodules are problematic. Their detection generates 2 years of CT scan follow-up, causing patient anxiety, immense cost and a high radiation burden. Few of these nodules are malignant. The 2005 Fleischner society guidelines are complex with no reference to the value of PET- CT imaging.

The Sheffield Teaching Hospital Trust follows a modified Fleischner protocol where the majority of patients have 3 follow-up scans over 2 years. This study aimed to:
- Determine the final diagnosis of each nodule and the number of follow-up CT scans performed.

- Asses the value of PET-CT nodule imaging.

- Determine whether an experienced chest radiologist could predict which nodules were malignant/ benign by CT characteristics alone.

Pulmonary nodules under review were extracted from the Trust lung cancer database. The final diagnoses were determined after $2+$ years of follow up. An experienced chest radiologist reviewed all the nodules, recording their impression of whether the nodules were benign/ malignant.

162 nodules in 140 patients were analysed. Six patients were excluded as no follow up data. $148 \backslash 156$ nodules were benign (95\%), 7 malignant and one presumed malignant (had inadequate follow-up). The 140 patients had 427 scans follow-up CT scans (mean 2.7, mode 3). 47 patients had fewer scans than required by protocol due to eg nodule resolution (9), recommendation to stop follow-up on CT report (13) and diagnosis of other disease (11). 20/22 patients had $4+$ follow-up scans due to shortened time interval between scans.

35 patients had PET CT scan. 28/29 nodules with low FDG uptake were morphologically benign and stable at 2 years. 1 "cold" nodule with malignant morphology was resected (adenocarcinoma).

The analysis of lung nodules by experienced chest radiologist found NPV 97.6\%, PPV 15\%, specificity $81 \%$ and sensitivity $62.5 \%$.

Comment The vast majority of lung nodules followed-up were benign. Better use of CT nodule morphology and review by an experienced chest radiologist is advised. We recommend that patients with a "cold", morphologically benign, nodule on PETCT scan should have a single 12 month CT scan to confirm stability.

\section{REFERENCES}

1. H MacMahon, et al. Radiology 2005;237:395-400.

\section{P50 A LOCAL ASSESSMENT OF THE ESCALATING IMPACT OF PULMONARY NODULE SURVEILLANCE AND ITS RELATIONSHIP TO PATIENT OUTCOMES IN A DGH}

LD Calvert, S Chatterji, F Miller, SO Brij, A Nakas; Peterborough City Hospital NHS Foundation Trust, Peterborough, UK

10.1136/thoraxjnl-2013-204457.200

Introduction Recent national emphasis on increased awareness to improve detection of early lung cancer has led to dramatic increases in CT chest scans being performed. Consequently, increased detection of incidental lung nodules requires monitoring. In response, we set up a dedicated Nodule Surveillance Service ( 2 physicians, 1 radiologist, 1 surgeon, 1 tracker). To determine likely future service requirements, we assessed current resource allocation to nodule surveillance and related this to patient outcomes.

Methods Patients discussed though our local Nodule Surveillance Service over 6 months were identified, and their electronic records reviewed.

Results 107 patients (64 male) undergoing surveillance were discussed November 2012-May 2013: 71 had single nodules, 36 multiple. This constituted an average 25 extra patient-discussions/month. Mean age 67years (range 39-93years); smoking status 27 current, 53 ex-smokers, 22 non-smokers. Referral 